\title{
Cuestionario Honey-Alonso de estilos de aprendizaje: Análisis de sus propiedades Psicométricas en Estudiantes Universitarios
}

\section{Honey-Alonso Learning Styles Questionnaire: An Analysis of its Psychometric Properties in College Students}

\author{
Agustín Freiberg Hoffman ${ }^{1} \quad$ Mercedes Fernández Liporace ${ }^{2}$ \\ Universidad de Buenos Aires, Argentina
}

(Rec: septiembre 2012 - Acep: diciembre 2012)

\begin{abstract}
Resumen
Se investigan propiedades psicométricas del Cuestionario Honey-Alonso de Estilos de Aprendizaje, mediante dos estudios de validez, uno de contenido y aparente, y otro de constructo. El primero examinó reactivos y escala mediante juicio experto y administraciones piloto. El segundo revisó la estructura dimensional mediante análisis de componentes principales (ACP) y consistencia interna por un lado; y análisis factorial confirmatoria (AFC) por el otro, a partir de dos muestras independientes de estudiantes universitarios argentinos. El primer estudio modificó 8 reactivos, y conservó la escala dicotómica de respuesta. Del segundo, el ACP extrajo cuatro dimensiones -convergente, asimilador, adaptador, pragmático- con adecuada consistencia interna, respondiendo sólo la pragmática al modelo teórico de partida. El AFC comparó el modelo del ACP con el de Alonso, Gallego y Honey (1994), obteniéndose un mejor ajuste del primero. Los resultados avalan la estructura factorial obtenida en el ACP concluyendo que el cuestionario evalúa los cuatro estilos hallados.

Palabras clave: CHAEA, estilos de aprendizaje, estudiantes universitarios, validez de constructo, validez de contenido.
\end{abstract}

\begin{abstract}
Honey-Alonso Learning Styles Questionnaire's psychometric properties were analyzed. In order to do this, two different studies were developed: content and facies validity in one hand, and construct validity procedures in the other. The very first examined items and scale by expert judgment. The second one used principal components analysis (PCA) and internal consistency in a preliminary stage, and confirmatory factor analysis (CFA) in the following. Two independent Argentinian college student samples were analyzed. Content validity results indicate the needing of modifying 8 items. Dichotomous answer scale was preserved. According to the results of construct validity, CPA showed four dimensions with better fit and good internal consistency. Only one of them -pragmatic component- responded to the original model. The remaining factors -assimilatory, convergent, adaptive- were identified as belonging to Kolb's (1984) theoretical model. Confirmatory factor analysis compared PCA model with Alonso, Gallego and Honey's model (1994), obtaining a better fit for the first. Results support PCA's structural dimensions for the questionnaire.
\end{abstract}

Key words: CHAEA, learning styles, college students, construct validity, content validity

\footnotetext{
Correspondencia a: Vallejos 2379, $4^{\circ}$ Piso, Código Postal: 1419, Ciudad Autónoma de Buenos Aires, Argentina. E-mail: afreiberg@psi.uba.ar.

Perteneciente también a Consejo Nacional de Investigaciones Científicas y Técnicas, CONICET, en Argentina.
} 


\section{Introducción}

El sistema educativo universitario local registra actualmente una baja en el rendimiento académico y un incremento de los índices de deserción, reflejando ello un empobrecimiento de los aprendizajes. Se estima que a nivel nacional el 58\% de la población universitaria de primer año abandona o cambia de carrera (Pintos, 2012), advirtiéndose en 2006 distintos niveles de deserción según las zonas, $20.2 \%$ en la Universidad de Buenos Aires, $62.8 \%$ en la de Jujuy, $53.2 \%$ en la Universidad de Santiago del Estero y $46 \%$ en la de la Patagonia Austral, con sede en Rio Gallegos (La Nación, 2008). Por su parte autoridades de distintas universidades nacionales indican que se gradúa aproximadamente el $25 \%$ de los ingresantes (Clarín, 2012), dato acompañado por las estadísticas oficiales de Argentina (Ministerio de Educación, 2009).

Distintas investigaciones (Núñez, Rodríguez, Fernández, Cerezo \& González, 2011; Cortés \& Palomar, 2008; Porcel, Dapozo \& López, 2010) dan cuenta de la influencia que poseen ciertas variables sobre el ejercicio académico de los estudiantes universitarios, estimando que su conocimiento permitiría por un lado predecirlo en alguna medida y por otro, planificar acciones dirigidas a mejorar los aprendizajes. A nivel local pueden hallarse estudios de variables sociodemográficas y académicas -tipo de educación secundaria media o técnica; orientación elegida; nivel educativo máximo alcanzado por la madre; aptitudes; razonamiento espacial, abstracto y verbal; velocidad y precisión; ortografía y leguaje; cálculo; estilos de pensamiento; capacidad educativa; y fortalezas y virtudes de carácter moral, entre otras (Corengia, Mesurado \& Redelico, 2006; Cosentino, Torres \& Clotet, 2009; González, Castro \& González, 2008; Vázquez y Noriega, 2011).

En esta línea, los estilos de aprendizaje constituyen otra variable relacionada con el rendimiento académico (Concha \& López, 2009; Drysdale, Ross \& Schulz, 2001; Luengo González \& González, 2005). Este constructo se define como un conjunto de atributos cognitivos, afectivos y fisiológicos que determinan el modo en que las personas perciben, interaccionan y responden a sus ambientes de aprendizaje (Keefe, 1988). Las diferentes configuraciones de estos rasgos dan lugar a los llamados estilos de aprendizaje (López Barajas \& Sarrate, 2002), que a su vez comprenden diversos procesos que, al conjugarse con el contexto, permiten realizar tareas de: exploración y emisión de respuestas; discriminación; selección y asimilación de información relevante y novedosa; y adaptación de conocimientos recientes a los ya adquiridos (Kolb, 1976). Si bien todos estos procedimientos cognitivos coexisten y se relacionan entre sí, es habitual que alguno se halle más acentuado, facilitando la adquisición de una clase de contenidos y dificultando la de otros (Kolb \& Kolb, 2005b). Dado que las distintas carreras universitarias demandan una combinación de estilos específica, aquellos que mejor se adapten a cada disciplina serán los más exitosos (Alonso, Gallego \& Honey, 1994; Kolb, 1984). Tales conclusiones han sido verificadas en estudios recientes realizados en otros contextos, evidenciándose el carácter predictivo de los estilos respecto del rendimiento estudiantil (Coloma, Manrique, Revilla \& Tafur, 2008; Esguerra \& Guerrero, 2010; Juárez, Hernández \& Escoto, 2011).

Esta relación entre estilos de aprendizaje y rendimiento académico motiva el interés del presente estudio por analizar las propiedades psicométricas de un instrumento dedicado a su evaluación. Se espera como fin ulterior poner a disposición de los profesionales del ámbito educativo universitario una herramienta que cuente con evidencias de validez y confiabilidad, y que permita ponderar los diferentes estilos para así, a partir de su descripción, facilitar la toma de decisiones tendientes a mejorar los procesos de aprendizaje.

\section{El modelo de Kolb}

Uno de los modelos más influyentes en este campo es el del aprendizaje experiencial de Kolb (1984). Su estructura, en sus aspectos generales, subyace a diversos instrumentos tales como el Learning Style Inventory (LSI) (Kolb, 1976), el Learning Styles Questionnaire (LSQ) (Honey \& Mumford, 1986), el Learning Type Measure (LTM) (McCarthy, 1987), el Cuestionario Honey-Alonso de Estilos de Aprendizaje (1994) y el Portafolio de Dimensiones Educativas (PDE) (Muñoz \& Silva, 2003).

Según esta propuesta teórica, la generación del conocimiento se define como un proceso de transformación de la experiencia percibida. Contempla dos dimensiones: percepción y procesamiento. La primera está vinculada a la captación y conceptualización de la experiencia, mientras que la segunda, se relaciona con el pensamiento y la comprobación de la información novedosa. Al interior de cada factor se encuentran dos procesos dialécticos o etapas que intervienen en toda instancia de aprendizaje, correspondiéndole a la dimensión perceptual los de experiencia concreta y 
conceptualización abstracta y a la procesual, la observación reflexiva y la experiencia activa (Kolb, 1984).

De acuerdo a Kolb \& Kolb (2009a), cada una de estas etapas reúne determinadas clases de comportamientos. La experiencia concreta (EC) comprende el entusiasmo por participar en nuevas prácticas, atendiendo al aquí y ahora y cambiando rápidamente de actividad ante la rutina y el aburrimiento.

La integración de datos empíricos con teorías lógicas y complejas es una conducta perteneciente al proceso de conceptualización abstracta (CA). Éste se caracteriza por el abordaje de los problemas mediante pasos preestablecidos y siempre de modo estructurado, acentuándose el perfeccionismo, la racionalidad, la objetividad y la aversión por la ambigüedad. La observación reflexiva (OR) se basa en una conducta predominantemente observacional y de recolección de información desde múltiples puntos de vista. La prudencia, el pensamiento y la inacción son algunas de sus cualidades destacadas. Los comportamientos típicos de la experimentación activa (EA) corresponden esencialmente a la veloz puesta en práctica de ideas, sin considerar su grado de verosimilitud. Se destaca una excelente capacidad para tomar decisiones generalmente acertadas, así como también para funcionar en ambientes desestructurados (Kolb \& Kolb, 2009a).

Estos cuatro procesos participan de un ciclo continuo que interviene en la adquisición de conocimientos nuevos. De este modo los sujetos asimilan la experiencia mediante la observación (EC), pasando luego a una fase de reflexión (OR) que más tarde les permitirá relacionar los contenidos nuevos con los preexistentes, desembocando en una tarea de conceptualización (CA). Finalmente, estos conceptos recientemente incorporados serán puestos a prueba mediante la experiencia, confirmándose su adecuación y determinándose su conservación, su modificación o su eliminación (EA). La información resultante de este procedimiento conformará el fundamento de un nuevo período, repitiéndose la secuencia ya descripta (Kolb \& Kolb, 2009b).

Un caso claro de esta sucesión de procesos podría representarse en el ámbito universitario del siguiente modo. Un alumno concurre a clase y percibe la información que transmite el docente (EC), desencadenándose un proceso reflexivo acerca de aquélla, ya en ese preciso instante, como así también una vez finalizada la clase (OR). Posteriormente el estudiante lee los apuntes sobre el tema, y a la par que sigue reflexionando, comienza un proceso de conceptualización, articulando estos datos novedosos con los ya conocidos (lecturas de apuntes previos, teorías ya aprendidas, contenidos de diversas asignaturas, entre otros) (CA). Finalmente, pone a prueba este conocimiento novedoso al momento de rendir el examen y verifica su adecuación a través de los resultados obtenidos reflejados en su calificación (EA). En caso que el alumno fracasara en la evaluación, podría concurrir a tutorías, orientación o clases particulares, lo que daría lugar a la repetición de la secuencia anterior. En cambio, si tuviera éxito, podría concluir la instancia de aprendizaje en ese mismo momento u optar por consultar posibles dudas, o bien pedir detalles al docente sobre la instancia evaluativa, lo que le llevaría también a reeditar la secuencia. Este ciclo de aprendizaje ideal no ocurre de manera similar en cada individuo ni en cada ocasión. Por otra parte los procesos mencionados no se dan en idénticas medidas en un mismo sujeto, configurándose el estilo predominante a partir de los procesos que se presentan de modo acentuado. Por tal motivo, Kolb (1984) describe cuatro estilos de aprendizaje que tienen lugar a partir de la interacción producida entre los cuatro procesos. Las modalidades de aprendizaje propuestas caracterizan a las personas como adaptadoras, convergentes, divergentes y asimiladoras (Figura 1).

Cada estilo implica aspectos cognitivos, comportamentales y relacionales subyacentes (Kolb \& Kolb, 2005a). Los individuos con predominio del estilo divergente (experiencia concreta - observación reflexiva) eligen conocer a partir de la experiencia concreta. Eso los lleva a interesarse considerablemente por la cultura y actividades que les aportan información. Aprecian el trabajo en grupo, son de mente abierta y contemplan múltiples puntos de vista sobre una

Figura 1.

Modelo bidimensional de estilos de aprendizaje de Kolb (1976) (Castaño Collado, 2004).

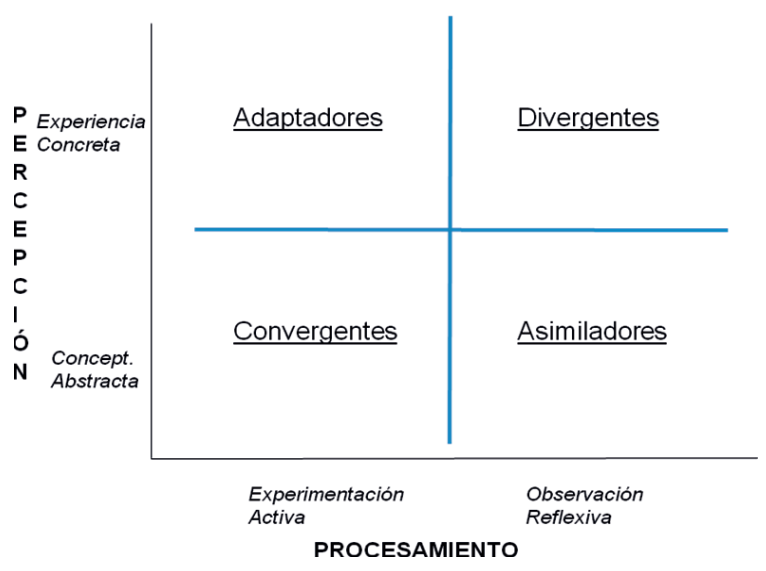


misma situación. Tienen gran habilidad para generar ideas novedosas, destacándose su enorme capacidad de retroalimentación.

El modo convergente (conceptualización abstracta - experimentación activa) parte de un conocimiento generado a través del uso de ideas y teorías. Se trata de personas poco permeables a recomendaciones ajenas, que disfrutan de la puesta a prueba de modelos novedosos, simulaciones y ensayos en laboratorios. Esto les hace preferir trabajar solos, resolviendo problemas técnicos y evitando toda actividad social que implique alguna clase de relación interpersonal (Kolb \& Kolb, 2005a).

Los asimiladores (conceptualización abstracta observación reflexiva) manejan y comprenden una amplia variedad de información. Son poco sociales y se concentran en ideas y conceptos abstractos. Focalizan su atención en la coherencia y validez lógica de las ideas más que en su utilidad práctica (Kolb \& Kolb, 2005a).

Finalmente el estilo adaptador (experiencia concreta - experimentación activa) describe a los adeptos a desafíos novedosos, que disfrutan de experimentar prescindiendo del análisis lógico de las situaciones. Se guían más por sus intuiciones y sentimientos, delegando los aspectos técnicos y confiando en el criterio de otros, lo que los hace apreciar el trabajo en equipo (Kolb \& Kolb, 2005a).

\section{Evaluación de los estilos de aprendizaje}

El Learning Style Inventory (LSI) (Kolb, 1976) toma como base para su construcción el modelo de Kolb, y tiene por objeto evaluar los estilos de aprendizaje en el ámbito escolar. Su revisión amplía el número de ítems de 9 a 12, dando lugar al LSI 2 (Kolb, 1985), retomada algo más tarde por Honey y Mumford (1986). Estos autores coinciden con la idea original sobre los cuatro procesos y su funcionamiento cíclico, presente en toda situación de aprendizaje. No obstante, a diferencia de Kolb, homologan procesos a estilos y los renombran como: activo, reflexivo, teórico y pragmático (Figura 2). De este modo, simplifican la estructura del modelo original al unificar el nivel de los estilos con el de los procesos.

En esta línea entonces, Honey y Mumford (1986) describen los estilos de aprendizaje a partir de las definiciones propuestas para los procesos por Kolb (1984), manteniendo la esencia de sus ideas originales intacta. De acuerdo con esta reformulación teórica diseñan el Learning Styles Questionnaire ( $L S Q$ ) para ser aplicado específicamente en el ámbito organizacional en el Reino Unido. Este cuestionario se compone de 80 ítems comportamentales (20 por cada estilo) de respuesta dicotómica (+ o -), a los que los examinados deben contestar según su acuerdo con cada afirmación.

Del LSQ y de su modelo surge el Cuestionario Honey-Alonso de Estilos de Aprendizaje -CHAEA(Alonso et al., 1994), como la versión adaptada (métrica, conceptual y lingüísticamente) para el ámbito académico español. Respeta tanto la configuración teórica propuesta por Honey y Mumford sobre los cuatro estilos, como también la estructura de la herramienta original en cuanto al número de ítems y a su modalidad de respuesta.

Figura 2.

Modelo Tetrafactorial de estilos de aprendizaje de Honey y Mumford (1986).

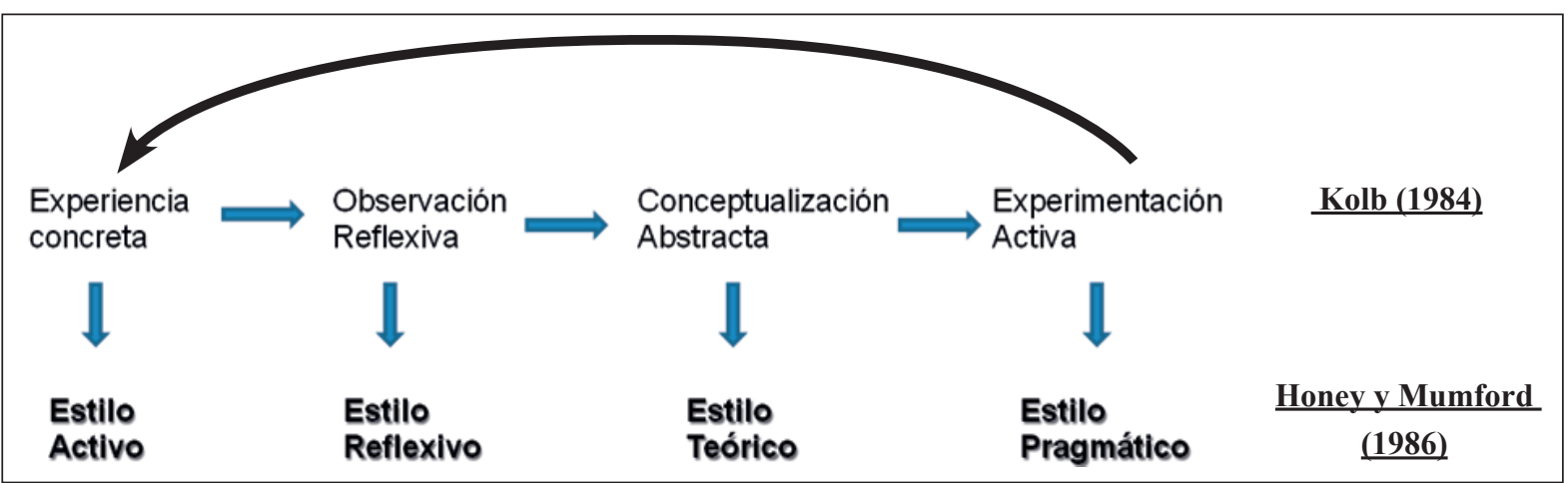




\section{Cuestionario Honey-Alonso de estilos de aprendizaje (CHAEA)}

Siguiendo los razonamientos antes descriptos, los análisis de calidad psicométrica del CHAEA parten de la hipótesis sustentada en el modelo, que supone cuatro estilos de aprendizaje (teórico, reflexivo, activo y pragmático) propuestos por Honey y Mumford (1986). Debido a que aquélla no consigue ser verificada mediante el análisis de componentes principales que extrae quince elementos, los autores acuerdan la solución de compromiso de agrupar los 20 ítems en las cuatro dimensiones según el diseño original (Alonso et al., 1994). Esta decisión genera una inconsistencia teórico-empírica que se refleja en un análisis correlacional entre estilos (hallándose fuertes asociaciones entre los modos reflexivo-teórico, teórico-pragmático, reflexivo-pragmático y activo-pragmático), llevando a Alonso et al., (1994) a argumentar que los factores no son excluyentes entre sí.

Pese a la debilidad estructural expuesta por sus diseñadores, este instrumento es retomado en adaptaciones regionales. Habiendo fracasado los variados intentos de reproducir la solución factorial propuesta por el modelo teórico, se consigue su replicación parcial en otras poblaciones latinoamericanas y españolas. Por caso, una investigación (Silva, 2009) con estudiantes universitarios brasileños de diferentes carreras (exactas y sociales) alcanza una solución de tres dimensiones de configuración mixta, componiéndose el factor 1 de ítems de los estilos activo y pragmático, el factor 2 de reflexivo y pragmático, y el factor 3 de reactivos teóricos y pragmáticos. Estas dimensiones son rotuladas como estilos activo, analítico y pragmático, respectivamente (Silva, 2009). Pasando ahora a desarrollos de habla hispana, dos investigaciones -una con alumnos españoles de pedagogía (Orellana, Bo, Belloch y Aliaga, 2002) y otra con estudiantes de ingeniería de la región de Nuevo Cuyo correspondiente a las provincias de Mendoza y La Rioja (Argentina) (Escalante, Barrionuevo y Mercado, 2009)- extrajeron dos componentes: teórico-reflexivo y activo-pragmático.

Asimismo, otro análisis realizado con alumnos universitarios de carreras sociales en Buenos Aires, da cuenta también de una estructura bifactorial que comprende el estilo teórico por una parte y el reflexivo por la otra, resultado atribuido a las características muestrales. Debido a que esta se compone de estudiantes de carreras ajenas a las ciencias exactas, tanto formales como fácticas, se estima que posiblemente la ausencia de los estilos activo y pragmático se asocie a esta escasa heterogeneidad de disciplinas (Freiberg, Uriel \& Fernández, 2011).

Dada la difusión del CHAEA como herramienta para la descripción de los estilos de aprendizaje en estudiantes universitarios, es que el presente trabajo se dirige a evaluar la calidad psicométrica del cuestionario, analizando finalmente el ajuste del modelo en alumnos y alumnas de nuestro medio. Debido al consenso teórico entre los autores que han trabajado en su construcción basándose en los cuatro estilos de aprendizaje descriptos (Alonso et al., 1994; Honey \& Mumford, 1986; Kolb, 1976; Kolb \& Kolb, 2005b), es que se intentará llevar a cabo un análisis que los reproduzca. Para responder a estos objetivos se realizaron estudios de validez de contenido y aparente por un lado, y de validez de constructo y consistencia interna por otro. El primero se abordó mediante juicio experto y estudios piloto. Para el segundo se aplicó análisis de componentes principales a fin de examinar la estructura latente del cuestionario en una muestra de estudiantes universitarios de Buenos Aires, esperándose una solución tetradimensional (estilos activo, teórico, reflexivo y pragmático), similar a la postulada por Alonso et al. (1994). En un segundo momento, se buscó confirmar la estructura aislada en el paso anterior en una muestra diferente a través de un análisis factorial confirmatorio.

Se pretende así poner a disposición de profesionales del ámbito educativo un instrumento breve que permita una rápida evaluación e identificación de los estilos implicados, en beneficio de los procesos de orientación vocacional y personal, así como también de tutorías o eventuales planificaciones de reformas pedagógicas, en pos de mejorar, indirectamente, los aprendizajes.

\section{Método}

\section{Diseño de investigación}

Se realizó un estudio ex post facto, correlacional de corte transversal (Nieto \& Rodríguez, 2010).

Participantes

Validez de contenido y aparente: 5 jueces expertos fueron convocados para la evaluación del contenido de los reactivos. Para realizar tres estudios piloto dirigidos a evaluar la comprensión lingüística de los reactivos -los dos primeros- y para valorar la pertinencia de la escala de respuesta a emplear-el tercero-participaron 8,20 y 20 alumnos. 
Validez de constructo: Se trabajó con dos muestras independientes de estudiantes universitarios de la Ciudad de Buenos Aires, una para el estudio factorial exploratorio y la otra para el confirmatorio.

- Estudio factorial exploratorio: 443 estudiantes (41.3\% varones y $58.7 \%$ mujeres). La distribución según sexo exhibió un porcentaje similar al informado por el Ministerio de Educación de la Nación Argentina (2009) para carreras universitarias (43.6\% varones y $56.4 \%$ mujeres). Las edades variaban entre 17 y 35 años $(\bar{X}=22.7$; D.T. $=3.32)$. Participaron sujetos provenientes de diversas facultades de instituciones públicas $(39.5 \%$ psicología, $34.3 \%$ ingeniería, $9.3 \%$ exactas, $8.4 \%$ medicina, $2.7 \%$ filosofía y letras, $3.8 \%$ artes musicales y sonoras).

- Análisis factorial confirmatorio: 682 estudiantes (39.1\% varones y $60.9 \%$ mujeres) de instituciones públicas $(94.6 \%)$ y privadas $(5.4 \%)$, cuyas edades se ubicaban entre 17 y 35 años $(\bar{X}=21.7$; D.T. $=3.32)$. Se incluyeron las siguientes facultades: $33.4 \%$ psicología, $22.3 \%$ ingeniería, $11.9 \%$ sociales, $10.6 \%$ medicina, $7 \%$ filosofía y letras, $6.9 \%$ exactas, $5.4 \%$ económicas, $2.5 \%$ artes musicales y sonoras.

La selección de instituciones y participantes fue intencional, simple e incidental. Conviene destacar que el predominio de alumnos de universidades públicas es consistente con la matriculación registrada a nivel nacional (Ministerio de Educación, 2009).

\section{Instrumentos:}

- Cuestionario Honey-Alonso de estilos de aprendizaje (CHAEA) (Alonso et al., 1994). Evalúa 4 estilos de aprendizaje -teórico, reflexivo, activo, pragmático- mediante 80 ítems (20 por cada dimensión) de respuesta dicotómica (+ o -) que el examinado debe responder según su acuerdo o desacuerdo con cada sentencia.

- Encuesta sociodemográfica y académica. Para caracterizar la muestra se recabaron datos sobre sexo, edad, tipo de universidad (pública o privada) y carrera en curso. Se recogió información adicional -grupo de convivencia, educación y profesión parental, y nivel socioeconómico autopercibido- a fin de valorar la validez interna relativa a la recolección de datos, eliminándose los casos en que hubiera más de cinco ítems sin responder en la escala, o bien se omitieran las respuestas de más de la mitad de los datos sociodemográficos y académicos, tomándose ello como indicador de falta de interés y de cooperación.

\section{Procedimientos:}

Validez de contenido y aparente: Se entregó a los jueces un instructivo con una breve reseña teórica conteniendo la descripción de cada estilo y las consignas para completar la tarea. Se brindó un cuadernillo con los 80 ítems ordenados aleatoriamente y una escala en la que los expertos debían indicar la dimensión a la que según su criterio pertenecía cada uno (Moreno Bayardo, 2000). Con los elementos resultantes se confeccionó una versión más breve del instrumento. Sobre ella se evaluó en primer término la comprensión de los reactivos, para lo que se administró el cuestionario a dos grupos independientes de alumnos en dos momentos, recogiéndose en la primera instancia una serie de recomendaciones sobre modificaciones lingüísticas, y comprobándose una adecuada inteligibilidad en la segunda aplicación. Posteriormente se valoró la pertinencia de la respuesta dicotómica con la colaboración de otro grupo de estudiantes. Se dividió el cuestionario en mitades, asignándoles, respectivamente, una modalidad dicotómica y una likert sin anclaje semántico. En la última la respuesta se presentó como un continuo donde sólo se especificaban los polos y el sujeto debía indicar mediante una cruz el punto del continuo donde hallara su preferencia. Se solicitó a los participantes responder ambas formas y completar al final, en una escala de 5 puntos, el grado de adecuación referido a cada modalidad de respuesta, donde 1 indicaba nada adecuado, 2 medianamente adecuado, 3 adecuado, 4 bastante adecuado y 5 extremadamente adecuado.

Validez de constructo: Se detallan los procedimientos de los estudios exploratorio y confirmatorio.

- Estudio factorial exploratorio: A fin de indagar la dimensionalidad del cuestionario éste se administró junto con una encuesta sociodemográfica y académica en el horario habitual de clases a cargo de un psicólogo habilitado y debidamente entrenado, con participación voluntaria, sin retribución económica y con consentimiento informado de los estudiantes. La confidencialidad de resultados y anonimato de los datos se aseguró en todo momento, informándose a los examinados que podrían dejar de responder en cualquier instancia de la aplicación.

- Análisis factorial confirmatorio: Con el objeto de analizar el ajuste de la estructura extraída en la fase exploratoria se aplicó la versión resultante de ese análisis a la segunda muestra de estudiantes. Se preservaron idénticas condiciones de administración para evitar efectos indeseables que alteraran la recolección de datos (Díaz, 2009). 
El estudio contó con los respectivos avales institucionales, así como con el de un comité de ética.

\section{Análisis de datos}

Validez de contenido y aparente: En relación al juicio experto, se tomó como criterio para la conservación de ítems que el número de jueces que hubiera acordado una misma clasificación no fuera inferior a 3 de los 5 convocados.

Para el estudio lingüístico de los ítems se estableció modificar aquellos cuyas recomendaciones hubieran sido señaladas al menos por 3 personas en el estudio piloto.

En relación a la elección de la escala de respuesta, se determinó que la adopción de una u otra dependiera de que al menos la mitad más uno de los sujetos hubieran evaluado como bastante adecuada (4) y extremadamente adecuada (5) una de ellas y simultáneamente la otra como nada adecuada (1), medianamente adecuada (2) o adecuada (3).

Validez de constructo: a continuación se describen los análisis realizados en los estudios exploratorio y confirmatorio.

- Estudio factorial exploratorio: Incluyéndose los ítems resultantes del estudio de validez de contenido y aparente se empleó el método de componentes principales con rotación Varimax, forzando la extracción a cuatro factores, intentando reproducir la estructura de Alonso, et al. (1994). El carácter dicotómico de los elementos planteaba algunas necesidades. Por una parte, era imperioso el cálculo de una matriz de correlaciones tetracóricas para llevar a cabo el análisis (Christoffersson, 1975; Richaud de Minzi, 2005), dado que se disponía de variables categóricas que suponen una respuesta latente continua (Muthén, 1988; Piera \& Seva, 1994). Así se apeló al programa SYSTAT 12 que brinda esa posibilidad (SYSTAT Software, 2010). Ante la dicotomía de la respuesta, la consistencia interna de las cuatro dimensiones aisladas debía examinarse mediante el coeficiente $K R 20$ (equivalente al Lambda 3 de Guttmann) (Martínez, 2005; Raykov \& Marcoulides, 2011). Para esto se utilizó el SPSS 21 (IBM Corporation, 2012).

- Análisis factorial confirmatorio: Se puso a prueba la estructura extraída en la fase exploratoria testeando la adecuación del modelo a los datos empíricos a partir de la consideración de varios índices de ajuste (Pérez, Chacón \& Moreno, 2000; Ribeiro, Maia, Prieto \& Almeida, 2000). Por otra parte se comparó ese modelo empírico extraído del análisis de componentes principales con el teórico propuesto por Alonso, Gallego y Honey (AGH). Aquí también la dicotomía exigía recaudos. Si bien el análisis factorial confirmatorio es un procedimiento diseñado fundamentalmente para el tratamiento de variables continuas, su aplicación en variables dicotómicas se considera legítima siempre y cuando se trabaje sobre matrices de correlaciones tetracóricas (Kline, 2005). Por ello se empleó el software LISREL 8.80 (Scientific Software International, 2006).Finalmente, este carácter categórico de las variables impidió asumir el supuesto de normalidad, aplicándose el método de estimación de mínimos cuadrados ponderados (WLS), que requiere una muestra amplia $(n>200)$ y analiza los datos bajo estas condiciones (Flora \& Curran, 2004).

\section{Resultados}

\section{Validez de contenido y aparente}

Se conservaron luego del juicio experto 53 de los 80 ítems originales distribuidos del siguiente modo: 11 estilo reflexivo, 16 teóricos, 8 activo, 18 pragmático.

Como resultado de la adecuación lingüística del cuestionario a nuestro medio se reformularon 8 ítems de los 53 sometidos a examen.

El análisis de la escala de respuesta mostró que el uso de una u otra era indiferente para los alumnos, ya que la mayoría puntuó del mismo modo ambas formas. Por esta razón se optó por conservar el formato dicotómico, tal como se presentara en el cuestionario original.

\section{Validez de constructo}

\section{Estudio factorial exploratorio y análisis de consistencia}

Como paso previo se estudió la homogeneidad de los 53 elementos en la matriz de correlación, eliminándose aquellos con índices inferiores a 40

En segundo término, siguiendo la propuesta de Alonso et al. (1994), se fijó la extracción a cuatro factores y se aplicó un análisis de componentes principales (ACP) con rotación Varimax y normalización Kaiser, observándose un moderado ajuste y balance entre el número de sujetos y de ítems $(\mathrm{KMO}=.721$ Test de Esfericidad de Bartlett: $X^{2}=1576.183 \mathrm{gl} ; 378$ sig<.01). 
Por otra parte, se eliminaron todos los reactivos cuya carga en un factor no hubiera superado el valor de .40 (Valderrey Sanz, 2010), así como también aquellos que presentaran saturaciones simultáneas por encima de 40 en dos o más factores (Vallejo Seco, 1992).

La estructura tetrafactorial aislada dio cuenta del $47 \%$ de la varianza total, explicando el Factor 1 el 13\%, el Factor 2 el $12.7 \%$, el Factor 3 el $11.6 \%$ y el Factor 4 el 9.7\%, conservándose un total de 28 ítems de los 53 analizados (Tabla 1). Como puede apreciarse, tres de las cuatro dimensiones presentaron una composición mixta, lo que hace que los factores extraídos respondieran parcialmente a los cuatro estilos propuestos por el modelo de Alonso et al. (1994). Se detalla a continuación la configuración de las dimensiones.

El Factor 1 se identificó con los estilos reflexivos (ítems 10, 31, 32, 63, 70) y teórico (ítems 4, 17, 33, 71 y 78$)$.

El Factor 2, al igual que el anterior, resultó una dimensión compuesta conformada por el estilo activo $(20,43,51)$ y el estilo pragmático $(30,52)$.

El Factor 3 advirtió también una composición mixta, con ítems del estilo teórico $(2,50,66,80)$ y del pragmático $(24,40,53,62)$.

Tabla 1.

CHAEA (Versión abreviada estudiantes universitarios de Buenos Aires). Estructura factorial.

\begin{tabular}{|c|c|c|c|c|}
\hline \multirow[b]{2}{*}{ ITEMS } & \multicolumn{4}{|c|}{ Componentes } \\
\hline & Teórico/Reflexivo (F1) & Activo/Pragmático (F2) & Teórico/Pragmático (F3) & Pragmático (F4) \\
\hline chaea04 & .703 & & & \\
\hline chaea10 & .528 & & & \\
\hline chaea17 & .479 & & & \\
\hline chaea31 & .658 & & & \\
\hline chaea32 & .54 & & & \\
\hline chaea33 & .552 & & & \\
\hline chaea63 & .546 & & & \\
\hline chaea 70 & .604 & & & \\
\hline chaea71 & .545 & & & \\
\hline chaea78 & .668 & & & \\
\hline chaea20 & & .79 & & \\
\hline chaea30 & & .633 & & \\
\hline chaea43 & & .566 & & \\
\hline chaea51 & & .817 & & \\
\hline chaea52 & & .864 & & \\
\hline chaea02 & & & .527 & \\
\hline chaea24 & & & .666 & \\
\hline chaea40 & & & .723 & \\
\hline chaea50 & & & .494 & \\
\hline chaea53 & & & .49 & \\
\hline chaea62 & & & .516 & \\
\hline chaea66 & & & .598 & \\
\hline chaea 80 & & & .607 & \\
\hline chaea14 & & & & .456 \\
\hline chaea68 & & & & .555 \\
\hline chaea 72 & & & & .879 \\
\hline chaea 73 & & & & .479 \\
\hline chaea76 & & & & .643 \\
\hline
\end{tabular}


El Factor 4 se integró exclusivamente con elementos del estilo pragmático $(14,68,72,73$ y 76).

Teniendo en cuenta la cercanía de la agrupación de los ítems con el modelo de Kolb (1984), en tres de los cuatro factores se decidió etiquetarlos según su criterio. De esta manera el Factor 1 coincidía con el estilo asimilador, compuesto por los estilos teórico y reflexivo, que en el modelo de Kolb representaban los procesos observación reflexiva y conceptualización abstracta.

El Factor 2 se identificó con el estilo convergente, conformado por los estilos teórico y pragmático, homologado a los procesos de conceptualización abstracta y experimentación activa de Kolb.

El Factor 3 se identificó con el estilo adaptador, constituido por los estilos activo y pragmático, coincidentes con los procesos de experiencia concreta y experimentación activa de Kolb.

A diferencia de las dimensiones anteriores, el Factor 4 respondió íntegramente al estilo pragmático propuesto por Alonso et al. (1994).

El análisis de la consistencia interna de cada una de las dimensiones se realizó a través del cálculo del coeficiente $K R 20$, obteniéndose un índice de .65 para el Factor 1, .63 para el Factor 2, .62 para el Factor 3 y .50 para el Factor 4.

\section{Estudio factorial confirmatorio}

El análisis comenzó teniendo en cuenta el coeficiente de determinación, que es un indicador de empleo poco convencional que permite indagar la relación entre los reactivos y las variables latentes (Kline, 2005). Este coeficiente se calculó para los 28 ítems en ambos modelos, a partir de las correlaciones establecidas entre cada uno de los indicadores y las variables latentes a las que se encuentran asociadas, permitiendo ponderar el aporte de cada reactivo a la explicación de la varianza. Los valores incluidos en la tabla 3 indican que 16 de los 28 ítems contribuían en mayor medida a la explicación de la varianza total del modelo empírico aislado en el ACP por sobre el AGH, mientras que 10 superaban en el modelo teórico AGH al del ACP. Sólo 2 ítems funcionaban de igual modo tanto en uno como en otro. Por otra parte si se toma como porcentual mínimo óptimo de varianza explicada que cada elemento debe aportar a su factor el valor de $50\left(\mathrm{R}^{2}=.50\right)(\mathrm{Kline}, 2005)$, puede observarse que 11 de los reactivos del modelo empírico ACP cumplían con esta regla, mientras que en el modelo teórico AGH sólo 7 alcanzaban este valor. Este análisis preliminar da cuenta de un mayor carácter explicativo de los elementos para con las variables latentes a favor del modelo ACP.

Tabla 2

Coeficientes de determinación.

\begin{tabular}{llll}
\hline \multicolumn{1}{c}{ Modelo ACP } & \multicolumn{1}{c}{ Modelo AGH } \\
\hline \multicolumn{1}{c}{ Ítems } & \multicolumn{1}{c}{$\mathrm{R}^{2}$} & \multicolumn{1}{c}{ Ítems } & $\mathrm{R}^{2}$ \\
\hline chaea02-Teo/Prag & .28 & chaea02-Teórico & .00 \\
chaea04-Teo/Ref & .42 & chaea04-Teórico & .66 \\
chaea10-Teo/Ref & .31 & chaea10-Reflexivo & .31 \\
chaea14-Pragmático & .23 & chaea14-Pragmático & .05 \\
chaea17-Teo/Ref & .52 & chaea17-Teórico & .53 \\
chaea20-Act/Prag & .64 & chaea20-Activo & .49 \\
chaea24-Teo/Prag & .27 & chaea24-Pragmático & .21 \\
chaea30-Act/Prag & .36 & chaea30-Pragmático & .36 \\
chaea31-Teo/Ref & .50 & chaea31-Reflexivo & .62 \\
chaea32-Teo/Ref & .38 & chaea32-Reflexivo & .48 \\
chaea33-Teo/Ref & .31 & chaea33-Teórico & .36 \\
chaea40-Teo/Prag & .62 & chaea40-Pragmático & .35 \\
chaea43-Act/Prag & .25 & chaea43-Activo & .20 \\
chaea50-Teo/Prag & .46 & chaea50-Teórico & .42 \\
chaea51-Act/Prag & .86 & chaea51-Activo & .90 \\
chaea52-Act/Prag & .86 & chaea52-Pragmático & .98
\end{tabular}




\begin{tabular}{llll}
\hline \multicolumn{1}{c}{ Modelo ACP } & & \multicolumn{1}{c}{ Modelo AGH } \\
\hline chaea53-Teo/Prag & .41 & chaea53-Pragmático & .10 \\
chaea62-Teo/Prag & .52 & chaea62-Pragmático & .08 \\
chaea63-Teo/Ref & .55 & chaea63-Reflexivo & .27 \\
chaea66-Teo/Prag & .58 & chaea66-Teórico & .29 \\
chaea68-Pragmático & .30 & chaea68-Pragmático & .19 \\
chaea70-Teo/Ref & .67 & chaea70-Reflexivo & .40 \\
chaea71-Teo/Ref & .28 & chaea71-Teórico & .55 \\
chaea72-Pragmático & .74 & chaea72-Pragmático & .37 \\
chaea73-Pragmático & .35 & chaea73-Pragmático & .20 \\
chaea76-Pragmático & .14 & chaea76-Pragmático & .18 \\
chaea78-Teo/Ref & .46 & chaea78-Teórico & .52 \\
chaea80-Teo/Prag & .38 & chaea80-Teórico & .00 \\
\hline
\end{tabular}

Figura 3.

Modelo CHAEA. Versión abreviada (Análisis de Componentes Principales).

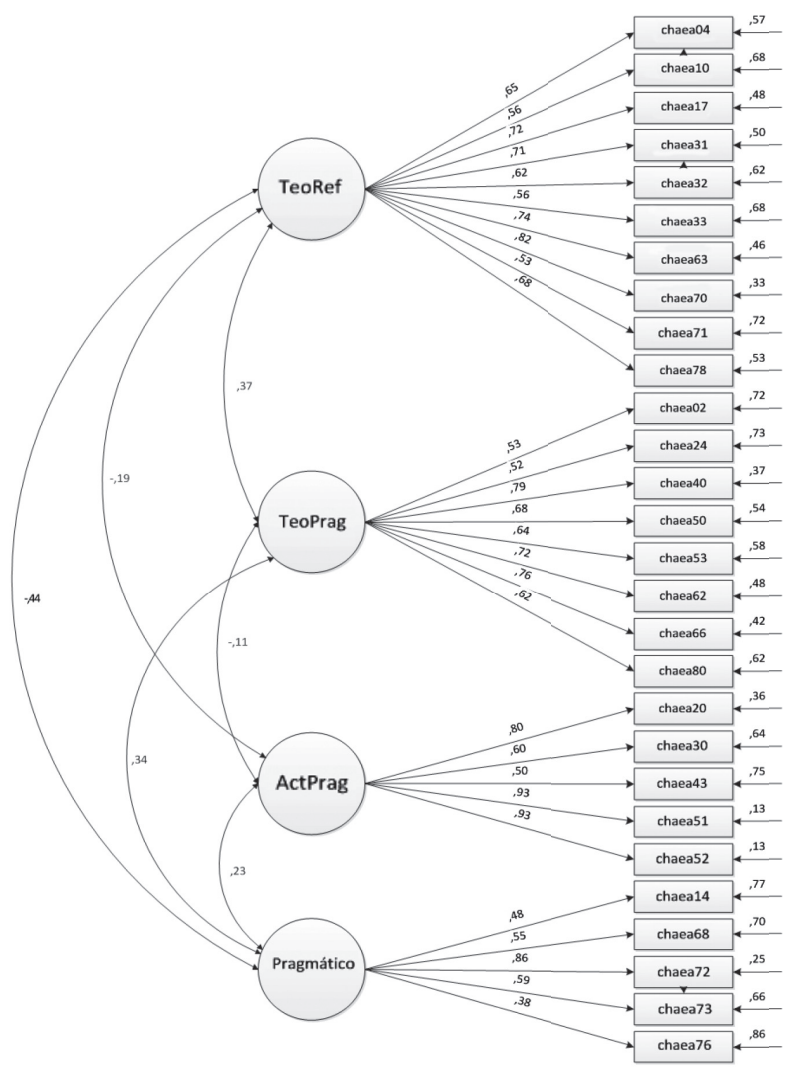

Figura 4.

Modelo CHAEA propuesto por Alonso, Gallego y Honey (1994).

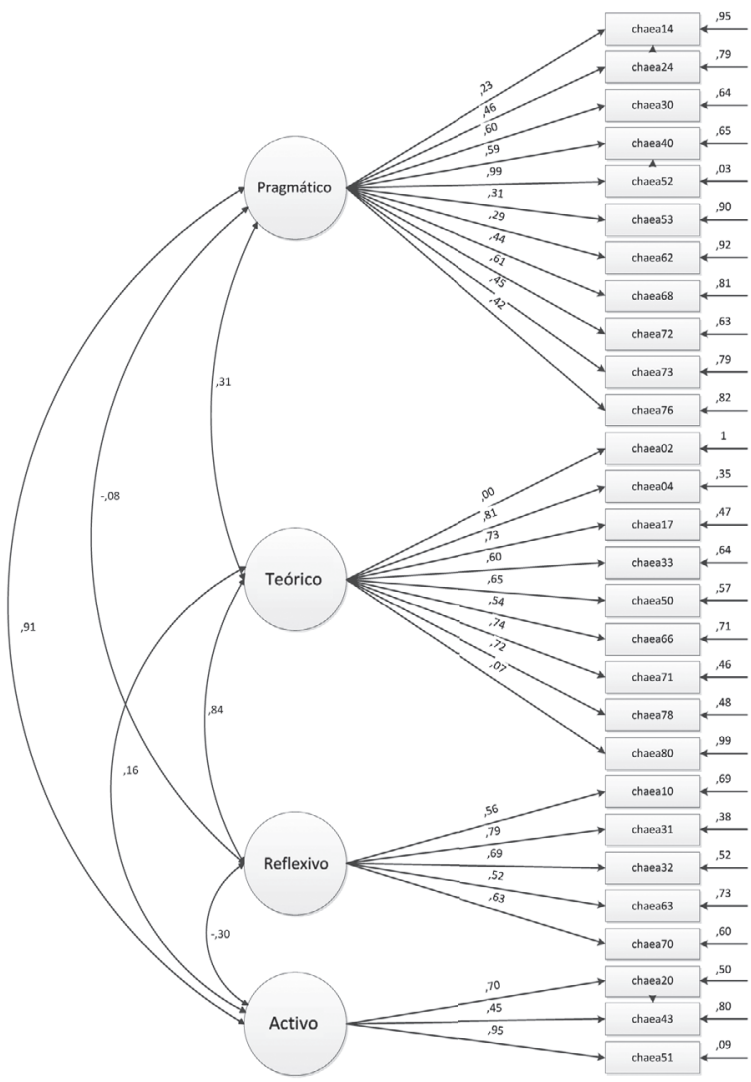


A continuación se comparó el ajuste de ambos modelos, a fin de estudiar la estructura interna de la escala (Figuras 3 y 4 ).

Se registró en ambas soluciones la presencia de casos Heywood (varianzas estimadas negativas), cuyos valores fueron conservados sin aplicar tratamiento estadístico especial, ya que su ocurrencia era esperable al trabajar con correlaciones tetracóricas y métodos de estimación libre asintótica (WLS) (Yuan, Wu \& Bentler, 2011). Por otra parte, estudios anteriores demostraron que estadísticos como chi cuadrado no se ven alterados significativamente por la aparición de estas ecuaciones infractoras (Chen, Bollen, Paxton, Curran \& Kirby, 2001).

El ajuste de los modelos se analizó mediante diversos índices de bondad de ajuste considerados característicos del software utilizado (LISREL 8.8) (Jöreskog \& Sörbom, 1999) y recomendados también por varios autores (e.g. Holgado, Chacón, Barbero \& Vila, 2010; Kline, 2005). De este modo, se tomaron en cuenta para la comparación los índices $\chi 2$, GFI (Goodness of Fit Index), AGFI (Adjusted Goodness of Fit Index) y RMSEA (Root Mean Square Error of Approximation). También se utilizaron los índices de parsimonia PGFI (Parsimony Goodness of Fit Index) y PNFI (Parsimony Normed Fit Index) (Schumacker \& Lomax, 2004).

Como puede notarse a partir del examen de la tabla 3, el modelo empírico extraído del ACP presentó índices de ajuste levemente superiores al modelo teórico AGH. Los valores GFI y AGFI en el modelo ACP fueron superiores a .90, valor mínimo consensuado como punto de corte entre un buen ajuste y uno mediocre (Byrne, 1998; Kelloway, 1998). En el modelo AGH sólo superó el valor de corte el índice GFI; no así el AGFI, que se ubicaba cerca del mismo.

Respecto de los valores de RMSEA puede decirse que un buen ajuste se sitúa entre 0 y .08 (Lévy \& González, 2006), guarismo que fue alcanzado por el modelo ACP, aunque no por el AGH.

Finalmente, considerando los índices de parsimonia (PGFI y PNFI) se advirtió que ambos valores resultaban superiores en el modelo ACP, indicando su mayor adecuación. Por otra parte, la diferencia de 08 entre los valores PNFI de ambos modelos, al ubicarse dentro de los valores mínimos (.06 a .09), permite afirmar la existencia de cambios sustanciales entre ellos (Lévy, Martín \& Román, 2006).

\section{Discusión}

La solución alcanzada en la fase exploratoria y luego confirmada con mejor ajuste (ACP) no consigue replicar de manera precisa los cuatro factores propuestos por Alonso et al. (1994) en el modelo AGH: teórico, reflexivo, activo y pragmático. Así se informa con mejores índices una estructura también tetrafactorial (ACP) con tres dimensiones de composición mixta (teórico-reflexivo, teórico-pragmático y activopragmático) y una simple (pragmático). Esta estructura, aún no reflejando exactamente los postulados teóricos que sustentan el CHAEA, sí guarda correspondencia lógica con el modelo previo que le diera origen, propuesto por Kolb (1984). Según éste los estilos convergente, divergente, asimilador y adaptador, surgen de la conjunción de los procesos conceptualización abstracta, observación reflexiva, experiencia concreta y experimentación activa, homologados por Alonso et al. (1994) en el AGH a los estilos teórico, reflexivo, activo y pragmático, respectivamente.

La lectura de los resultados a la luz del modelo de Kolb permite afirmar que la combinación de ítems (producto de los análisis) que representan los diferentes procesos, da lugar a tres de los cuatro estilos: asimilador (teórico-reflexivo), convergente (teórico-pragmático) y adaptador (activo-pragmático). La cuarta dimensión está compuesta íntegramente por elementos del estilo pragmático formulado por Alonso et al. (1994) en el modelo AGH, nominado por Kolb (1984) como experimentación activa. Para este último se decide por razones teóricas, conservar la nomenclatura otorgada por los autores del CHAEA,

Tabla 3

CHAEA. Comparación de ajustes entre modelos.

\begin{tabular}{cccccccc}
\hline & \multicolumn{9}{c}{ Índices de Ajuste } & \multicolumn{3}{c}{ Índices de Parsimonia } \\
\hline \multirow{3}{*}{ Modelo ACP } & $\chi^{2}$ & $\mathrm{gl}$ & GFI & AGFI & RMSEA[IC]* & PGFI & PNFI \\
Modelo AGH & $1668,341^{* *}$ & 344 & .934 & .922 & $.075[.071-.078]$ & .792 & .669 \\
& $2240,242^{* *}$ & 344 & .912 & .896 & $.09[.086-.093]$ & .772 & .587 \\
\hline
\end{tabular}


por corresponder el término experimentación activa al nivel de los procesos.

Es importante destacar que a pesar de estas distinciones -que también pueden entenderse como solapamientos en los constructos de partida-, la estructura tetrafactorial resultante del presente estudio guarda coherencia teórica y práctica, asociada a la evolución de los modelos y de los instrumentos. El cuestionario sometido a análisis parte de un supuesto que suprime uno de los niveles planteados por Kolb al homologar los estilos a los procesos. Tanto Honey y Mumford (1986) como Alonso et al. (1994) en el modelo AGH construyeron sus instrumentos (LSQ y CHAEA, respectivamente) en base a esta asunción, planteando cuatro estilos (teórico, reflexivo, activo y pragmático). Si bien los teóricos del CHAEA no consiguieron aislar mediante la factorización exploratoria las cuatro dimensiones propuestas, sí obtuvieron a partir de un análisis correlacional asociaciones significativas entre estilos. Estas relaciones halladas por Alonso et al. (1994) coinciden por un lado con los datos correlacionales expuestos por Honey y Mumford (1986), y por otro con la agrupación de los elementos en los factores extraídos en el presente análisis (Tabla 4).

Así, el modo en que se relacionan y se agrupan los estilos antes mencionados encuentra su explicación en el modelo teórico de Kolb, que subyace a la teoría de Honey y Mumford y por contigüidad, a la de Alonso et al. (modelo AGH).

Resumiendo entonces, este trabajo no ha conseguido extraer y confirmar los factores del CHAEA, a excepción de la dimensión pragmática, ajustándose mejor a la propuesta de Kolb, de la que consigue reproducir tres de los cuatro estilos (convergente, asimilador, adaptador), desapareciendo el estilo divergente.

Por otra parte, se destaca el hecho de que los resultados obtenidos se asemejen a los reportados por otras investigaciones. Los autores referidos en párrafos anteriores, cuyas extracciones consiguieron replicar sólo de modo parcial la estructura original, arribando a dimensiones mixtas (Escalante, Barrionuevo \& Mercado, 2009; Orellana, Bo, Belloch \& Aliaga, 2002; Silva, 2009). No habían tomado en cuenta, probablemente, la factibilidad de realizar una lectura de los resultados desde el modelo de Kolb. Quizá por esta razón no señalaron la semejanza de sus soluciones con los estilos de este autor.

La adaptación del CHAEA a nuestra población de estudiantes universitarios permite evaluar, así, cuatro estilos de aprendizaje: tres pertenecientes al modelo de Kolb (asimilador, convergente y adaptador), y uno propuesto por Alonso et al. en el modelo AGH (estilo pragmático).

Con respecto a los índices de consistencia de las dimensiones, inferiores al valor óptimo $(K R 20=.80)$, pueden aceptarse si se tiene en cuenta el escaso número de ítems que se conserva en cada una (Martínez, 2005). Dada la adecuada amplitud y heterogeneidad de la muestra recogida permite, por un lado, aumentar la precisión en las estimaciones por reducción del porcentaje de error y, por otro, incrementar el grado de confianza o probabilidad de las estimaciones efectuadas (Chitarroni, 2008), lo que puede proponerse como alternativa para mejorar la confiabilidad de los factores, la generación de ítems nuevos con el fin de extender la cantidad de reactivos por dimensión. Si bien esta opción es avalada por diversos autores (Furr \& Bacharach, 2008; Kaplan \& Saccuzzo, 2008; Kline, 1986), también se destaca que un elevado número de elementos acarrea dificultades asociadas al tiempo prolongado de administración. Versiones extensas de los instrumentos son propensas a generar errores aleatorios provenientes de los examinados causados por fatiga, aburrimiento, cansancio, entre otras posibilidades, que alteran los

Tabla 4

Índices de correlación entre estilos según modelo. Comparación con estructura extraída del ACP.

\begin{tabular}{lccc}
\hline & $\begin{array}{c}\text { Honey y Mumford } \\
(1986)\end{array}$ & $\begin{array}{c}\text { Alonso Gallego y } \\
\text { Honey (1994) }\end{array}$ & $\begin{array}{c}\text { Factores Análisis Componentes Principales } \\
\text { (Estilos según Kolb) }\end{array}$ \\
\hline Reflexivo-Teórico & .71 & .51 & Factor 1 (Asimilador) \\
Teórico-Pragmático & .54 & .39 & Factor 2 (Convergente) \\
Activo-Pragmático & .30 & .19 & Factor 3 (Adaptador) \\
Reflexivo-Pragmático & .42 & .20 & Relación no reflejada por los análisis \\
\hline
\end{tabular}


Figura 5.

Resumen de la estructura dimensional de los modelos de Kolb, AGH y ACP.

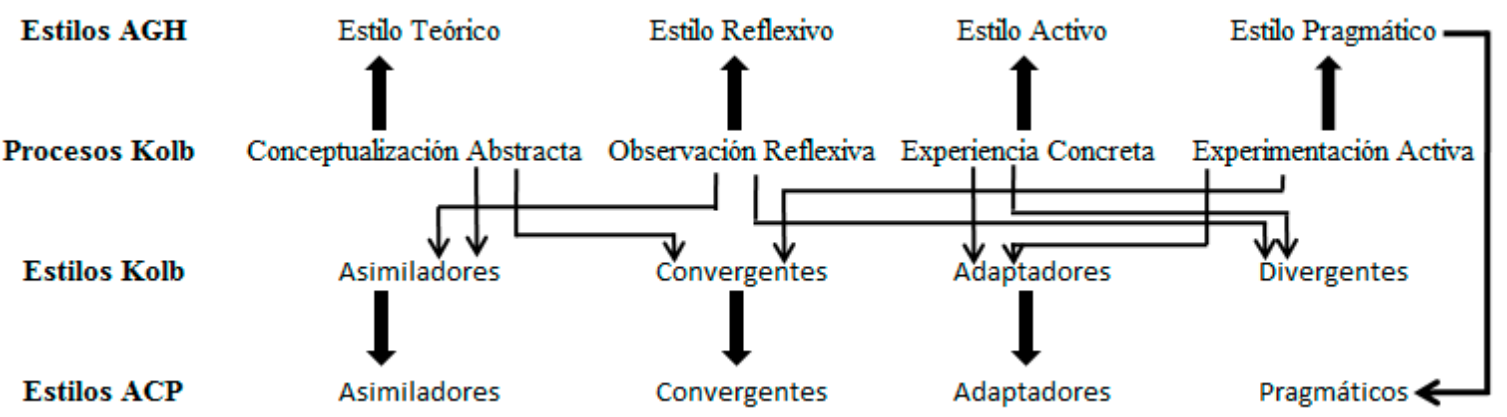

resultados debido a la distorsión de respuestas por contestaciones al azar, inadecuada interpretación de contenidos e inatención, entre otros.

Una limitación del estudio consiste en la imposibilidad de efectuar un análisis confirmatorio para comparar los modelos ACP y AGH con el propuesto por Kolb. La incompatibilidad entre el diseño del instrumento y la teoría de Kolb torna la comparación entre modelos una tarea meramente intuitiva, signada por la dificultad para reconstruir la dimensión divergente, debido a que inferir qué reactivos integrarían sus procesos (experiencia concreta y observación reflexiva) se vuelve un ejercicio especulativo.

Pese a la amplitud y heterogeneidad muestral señalada, otra limitación radica en la desigual proporción por carrera en las muestras analizadas, restricción que perjudica el estudio exploratorio ya que sus resultados, a diferencia del AFC, dependen fundamentalmente de esa variabilidad muestral (Byrne, 2001). La equiparación de proporciones según carreras podría influir en la extracción de factores del ACP permitiendo soluciones con ajustes potencialmente mejores. No obstante es importante destacar que esta dificultad, al no alterar el procedimiento confirmatorio tampoco modifica la validez de los resultados alcanzados.

Este análisis de calidad psicométrica ha dado lugar a una versión abreviada del CHAEA, con aceptables propiedades psicométricas, lingüísticamente adaptada a modismos locales, dirigida a valorar los estilos de aprendizaje confirmados -convergente, asimilador, adaptador, pragmático- en el ámbito educativo. Su fundamentación teórica responde al modelo de Kolb (1984) y en menor medida al de Alonso et al. (1994), posibilitando una interpretación aplicada de sus resultados.
Quizá la mayor contribución de este trabajo consista en la confirmación de tres de las cuatro dimensiones de Kolb (1984), que se validan a partir de evidencia empírica. La compleja agrupación de los reactivos obtenida en los resultados fortalece este modelo, dado que admite la posibilidad de que su estructura dimensional sea subyacente a otro instrumento (CHAEA), aparte del LSI, originalmente elaborado por Kolb (1976).

Se espera, en un futuro inmediato, concretar estudios que aporten evidencias de validez predictiva relativa a los estilos de aprendizaje según el rendimiento académico, así como también investigaciones que permitan identificar estilos distintivos en cada carrera universitaria. Disponer de esta información estimulará acciones ulteriores orientadas a mejorar los aprendizajes de los estudiantes universitarios, de cara a la disminución del fracaso y la deserción académica.

\section{Agradecimiento}

Este estudio se enmarca en una investigación subsidiada por la Universidad de Buenos Aires, y presenta el avance parcial de una beca de doctorado otorgada por esa casa de estudios.

\section{Referencias}

Alonso, C.M., Gallego, D.J., \& Honey, P. (1994). Los estilos de aprendizaje. Procedimientos de diagnóstico y mejora. Bilbao: Mensajero.

Bernardo, A.B., Núñez, J.C., Rodríguez, C., Bernardo, I., Fernández, E., Cerezo, R., \& González, A. (2011). Variables predictoras del rendimiento académico en el EEES. Revista d'Innovació Docent Universitària, 3, 26-34. 
Byrne, B.M. (1998). Structural Equation Modeling with LISREL, PRELIS and SIMPLIS. London: Lawrence Erlbaum Associates, Inc.

Byrne, B.M. (2001). Structural Equation Modeling with AMOS: Basic Concepts, Applications and Programming. London: Lawrence Erlbaum Associates, Inc.

Castaño, G. (2004). Independencia de los estilos de aprendizaje de las variables cognitivas y afectivo motivacionales. Tesis de Doctorado. Universidad Complutense de Madrid, Facultad de Psicología, España.

Chen, F., Bollen, K., Paxton, P., Curran, P., \& Kirby, J. (2001). Improper solutions in structural equation models. Sociological, Methods \& Research, 29(4), 468-508.

Chitarroni, H. (2008). La selección de la evidencia empírica. Universos y muestras. En: H. Chitarroni, La investigación en ciencias sociales: lógicas, métodos y técnicas para abordar la realidad social. (pp. 225-248). Buenos Aires: Universidad del Salvador.

Christoffersson, A. (1975). Factor analysis of dichotomized variables. Psychometrika, 40(1), 5-32.

Clarín (2012, 26 de agosto). Decanos y rectores reconocen que la alta deserción es un punto débil. Clarín.com, Sociedad. Recuperado de http://www.clarin.com/sociedad/educacion/Decanos-rectoresreconocen-desercion-punto_0_542945805.html

Coloma, C.R., Manrique, L., Revilla, D.M., \& Tafur, R. (2008). Estudio descriptivo de los estilos de aprendizaje de docentes universitarios. Revista Estilos de Aprendizaje, 1(1), 124-142.

Concha, S., \& López, B. (2009). Estilos de aprendizaje y rendimiento teórico-práctico de alumnos de imagenología, tercer año de odontología. Int. J. Odontostomat, 3(1), 23-28.

Corengia, A., Mesurado, B., \& Redelico, F. (2006). Las aptitudes educacionales y su relación con el rendimiento académico y la deserción: un estudio exploratorio. Revista Internacional de Estudios en Educación, 6(2), 75-83.

Cortés, A., \& Palomar, J. (2008). El proceso de admisión como predictor del rendimiento académico en la educación superior. Universitas Psychologica, 7(1), 199-215.

Cosentino, A.C., Torres, J.A., \& Clotet, C.I. (2009). El carácter moral predice el rendimiento académico. Memorias del I Congreso Internacional de Investigación y Práctica Profesional en Psicología. XVII Jornadas de Investigación de la Facultad de Psicología. V Encuentro de Investigadores en Psicología del Mercosur, 1, 419-420.

Díaz, A. (2009). Diseño Estadístico de Experimentos (2a. ed.). Medellín: Editorial Universidad de Antioquia.

Drysdale, M.T.B., Ross, J.L., \& Schulz, R.A. (2001). Cognitive learning styles and academic performance in 19 first-year university courses: Successful students versus students at risk. Journal of Education for Students Placed at Risk, 6(3), 271-289.

Escalante, E., Barrionuevo, R., \& Mercado, M. (2009). Aplicación de técnicas multidimensionales al estudio de los estilos de aprendizaje en estudiantes de ingeniería de la Universidad Tecnológica Nacional (UTN) de Mendoza y La Rioja. Cognición, 5(21), 34-42.

Esguerra, G., \& Guerrero, P. (2010). Estilos de aprendizaje y rendimiento académico en estudiantes de psicología. Revista Diversitas - Perspectivas en Psicología, 6(1), 97-109.

Flora, D., \& Curran, P. (2004). An empirical evaluation of alternative methods of estimation for confirmatory factor analysis with ordinal data. Psychological Methods, 9(4), 466-491.

Freiberg, A., Uriel, F., \& Fernández, M.M. (2011). Estudio factorial del cuestionario Honey-Alonso de estilos de aprendizaje (CHAEA) en estudiantes universitarios de Buenos Aires. Poster No230 presentado en el VIII Congreso Iberoamericano de Evaluación Psicológica. Lisboa, Portugal.

Furr, R.M. \& Bacharach V.R. (2008). Psychometrics. California: Sage Publications, Inc.
González, G., Castro Solano, A., \& González, F. (2008). Perfiles aptitudinales, estilos de pensamiento y rendimiento académico. Anuario de Investigaciones de la Facultad de Psicología de la Universidad de Buenos Aires, 15, 33-41.

Holgado, F., Chacón, S., Barbero, I., \& Vila, E. (2010). Polychoric versus Pearson correlation in exploratory and confirmatory factor analysis of ordinal variables. Quality and Quantity, 44(1), 153-166.

Honey, P., \& Mumford, A. (1986). The Manual of Learning Styles. Maidenhead, Berkshire: P. Honey, Ardingly House.

IBM Corporation (2012). IBM SPSS Statistics (21). Recuperado de http://www-01.ibm.com/software/es/analytics/spss/

Jöreskog, K.G., \& Sörbom, D. (1999). LISREL 8: user's reference guide. Lincolnwood, IL: Scientific Software International, Inc.

Juárez, C.S., Hernández-Castro, S.G., \& Escoto, M.C. (2011). Rendimiento académico y estilos de aprendizaje en estudiantes de psicología. Revista Estilos de Aprendizaje, 7(7), 79-92.

Keefe, J.W. (1988). Profiling and Utilizing Learning Style. Reston, Virginia: NASSP.

Kaplan, R.M. \& Saccuzzo, D.P. (2008). Psychological Testing. California: Wadsworth.

Kline, P. (1986). A handbook of test construction. New York: Methuen \& CO.

Kline, R. B. (2005). Structural equation modeling. New York: Guilford Press.

Kelloway, E.K. (1998). Using LISREL for Structural Equation Modeling. New Delhi : Sage Publications, Inc.

Kolb, A., \& Kolb, D. (2005a). Learning styles and learning spaces. Enhancing experiential learning in higher education. Academy of Management Learning \& Education, 4(2), 193-212.

Kolb, A., \& Kolb, D. (2005b). The Kolb learning style inventory - version 3.12005 technical specifications. HayGroup. Recuperado de www.whitewater-rescue.com/support/pagepics/lsitechmanual.pdf

Kolb, A., \& Kolb, D. (2009a). The learning way: Meta-cognitive aspects of experiential learning. Simulation Gaming, 40(3), 297-327.

Kolb, A., \& Kolb, D. (2009b). Experiential learning theory: A dynamic, holistic approach to management learning, education and development (pp. 1-59). En: S. Armstrong and C. Fukami (Eds.), The SAGE handbook of management learning, education and development. California: SAGES Publications.

Kolb, D. (1976). Learning Style Inventory: Technical Manual. Boston: McBer and Company.

Kolb, D. (1984). Experiential learning: experience as the source of learning and development. New Jersey: Prentice Hall, Inc., Englewood Cliffs.

Kolb, D. (1985). Learning Style Inventory: Technical Manual. Boston: HayGroup, Hay Resources Direct.

La Nación (2008). Causas del fracaso en la universidad. Recuperado de http://www.lanacion.com.ar/nota.asp?nota_id=1003217

Lévy, J.P., \& González, N. (2006). Modelización y Causalidad. En: J.P. Lévy y J. Varela (Eds.), Modelización con estructuras de covarianzas en ciencias sociales (pp. 155-175). Coruña: Netbiblo.

Lévy, J.P., Martín, M.T., \& Román, M.V. (2006). Optimización según estructuras de covarianza. En: J.P. Lévy \& J. Varela (Eds.), Modelización con estructuras de covacianzas en ciencias sociales (pp. 11-30). Coruña: Netbiblo.

López, E., \& Sarrate, M.L. (2002). La educación de personas adultas. Madrid: Dykinson

Luengo, R., \& González, J.J. (2005). Relación entre los estilos de aprendizaje, el rendimiento en matemáticas y la elección de asignaturas optativas en alumnos de E.S.O. Revista Electrónica de Investigación y Evaluación Educativa, 11(2). Recuperado de http://www.uv.es/RELIEVE/v11n2/RELIEVEv11n2_4.htm.

Martínez, R. (2005). Psicometría: teoría de los tests psicológicos y educativos. Madrid: Síntesis. 
Ministerio de Educación, Secretaría de Políticas Universitarias. (2009). Anuario 2009. Estadísticas Universitarias. Recuperado de http://repositorio.educacion.gov.ar:8080/dspace/bitstream/ handle/123456789/66203/Anuario-2009.pdf?sequence $=1$

McCarthy, B. (1987). The 4MAT System. Teaching to learning styles with right/left mode techniques. Revise Edition. Oak Brook, IL: Excel, Inc.

Moreno, M.G. (2000). Introducción a la Metodología de la Investigación Educativa 2. México, D.F.: Editorial Progreso.

Muñoz, B., \& Silva, C. (2003). Four dimensions to induce learning: the challenge profile (IESE Working Paper $N^{o} D / 512$ ). Recuperado de University of Navarra, IESE Business School website: http:// www.iese.edu/research/pdfs/DI-0520-E.pdf

Muthén, B. (1988). Testing the assumptions underlying tetrachoric correlations. Psychometrica, 53(4), 563-578.

Nieto, S., \& Rodríguez, J.M. (2010). Investigación y Evaluación Educativa en la Sociedad del Conocimiento. Salamanca: Ediciones Universidad de Salamanca.

Orellana, N., Bo, R., Belloch, C., \& Aliaga, F. (2002). Estilos de aprendizaje y utilización de las TIC en la enseñanza superior. Actas de la Conferencia Internacional sobre Educación, Formación y Nuevas Tecnologías. Virtual Educa 2004, Valencia 117. Recuperado de http://www.uv.es/ bellochc/doc\%20UTE/VE2002_117.pdf

Pérez, J.A., Chacón, S., \& Moreno, S. (2000). Validez de constructo: el uso del análisis factorial exploratorio-confirmatorio para obtener evidencias de validez. Psicothema, 12(2), 442-446.

Piera, P.J., \& Seva, U.L. (1994). Recuperación de la solución factorial a partir de variables dicotomizadas. Psicothema, 6(3), 483-491.

Pintos, M.E. (2012, 26 de marzo). En el primer año, el 58\% de los estudiantes dejan o cambian de carrera. Clarín.com, Sociedad, Recuperado de http://www.clarin.com/sociedad/primer-estudiantesdejan-cambian-carrera_0_670732977.html

Porcel, E.A., Dapozo, G.N., \& López, M.V. (2010). Predicción del rendimiento académico de alumnos de primer año de la FACENA
(UNNE) en función de su caracterización socioeducativa. Revista Electrónica de Investigación Educativa, 12(2), 1-21.

Raykov, T., \& Marcoulides, G. (2011). Introduction to Psychometric Theory. New York: Taylor \& Francis Group.

Ribeiro, I.S., Maia, J., Prieto, G., \& Almeida, L.S. (2000). Validez estructural de las pruebas de evaluación de realización cognitiva. Psicothema, 12(1), 137-143.

Richaud de Minzi, M.C. (2005). Desarrollos del análisis factorial para el estudio de ítem dicotómicos y ordinales. Revista Interdisciplinaria, 22(2), 237-251.

Schumacker, R.E., \& Lomax, R.G. (2004). A beginner's guide to estructural equation modeling (2a ed.). New Jersey, London: Lawrence Erlbaum Associates.

Scientific Software International (2006). LISREL (8.80). Recuperado de http://www.ssicentral.com/lisrel/resources.html

Silva, E. (2009). Estilos de aprendizagem em universitários brasileiros. Estabelecimento de perfis por titulação. Tradução, adaptação e análise do CHAEA (Tesis Doctoral). Recuperado de https://buleria.unileon.es/xmlui/bitstream/handle/10612/999/2009SILVA\%20 FALCHETTI\%2c\%20EDNA.pdf?sequence $=1$

SYSTAT Software (2010). SYSTAT (12). Recuperado de http://www. systat.com/SystatProducts.aspx

Valderrey Sanz, P. (2010). SPSS 17. Extracción del conocimiento a partir del análisis de datos. Madrid: RA-MA.

Vallejo Seco, G. (1992). Análisis Multivariantes Aplicados a las Ciencias del Comportamientno. Oviedo: Universidad de Oviedo.

Vázquez, S.M., \& Noriega, M. (2011). Razonamiento espacial y rendimiento académico. Revista Interdisciplinaria, 28(1), 145-158.

Yuan, K., Wu, R., \& Bentler, P. (2011). Ridge structural equation modeling with correlation matrices for ordinal and continuous data. Br J Math Psychol. Author Manuscript; Available in PMC 2011. Recuperado de http://www.ncbi.nlm.nih.gov/pmc/articles/ PMC3103762/ 